\title{
THE VARIANCE OF THE NUMBER OF ZEROS OF A STATIONARY NORMAL PROCESS ${ }^{1}$
}

\author{
BY M. R. LEADBETTER AND J. D. CRYER \\ Communicated by J. Doob, January 5, 1965
}

Let $x(t)$ be a separable stationary normal stochastic process with zero mean, covariance function $r(\tau)$ (with $r(0)=1$, for convenience), and spectrum $F(\lambda)$ having an absolutely continuous component. Let $N$ denote the number of times $x(t)$ crosses the zero level in $0 \leqq t \leqq T$ and write $\lambda_{2}$ for the second spectral moment $\int_{0}^{\infty} \lambda^{2} d F(\lambda)=-r^{\prime \prime}(0)$. Then it is well known that the mean of the random variable $N$ is given by $T \sqrt{ } \lambda_{2} / \pi$ (and, in fact, it has been recently shown by Ylvisaker [4] that this is true whether or not $\lambda_{2}$ is finite, provided $x(t)$ has continuous sample functions with probability one). The second moment has been given by a number of authors (e.g., [2], [3]) but the best conditions available to date include the existence of a sixth derivative for the covariance function $r(\tau)$.

Here we give the following result for the second moment of $N$ and indicate briefly the general lines of proof. Full details will appear elsewhere.

TheOREM. Suppose that $\lambda_{2}<\infty$ and that, for all sufficiently small $\tau$, $\lambda_{2}+r^{\prime \prime}(\tau) \leqq \Psi(\tau)$, where $\Psi(\tau)$ decreases as $\tau$ decreases to zero and is such that $\Psi(\tau) / \tau$ is integrable over $[0, T]$. Then

(1) $\varepsilon\left\{N^{2}\right\}=\varepsilon\{N\}+\iint_{0}^{T} d s d t \iint_{-\infty}^{\infty}|x y| p_{t-s}(0,0, x, y) d x d y$,

where $p_{\tau}(u, v, x, y)$ is the four-variate normal density for $x(0), x(\tau)$ and the (mean square) derivatives $x^{\prime}(0), x^{\prime}(\tau)$. That is, $p_{r}$ has the normal form with zero means and covariance matrix

$$
\Sigma=\left[\begin{array}{cccc}
1 & r(\tau) & 0 & r^{\prime}(\tau) \\
r(\tau) & 1 & -r^{\prime}(\tau) & 0 \\
0 & -r^{\prime}(\tau) & \lambda_{2} & -r^{\prime \prime}(\tau) \\
r^{\prime}(\tau) & 0 & -r^{\prime \prime}(\tau) & \lambda_{2}
\end{array}\right]
$$

(1) can be evaluated explicitly to yield

${ }_{1}^{1}$ Research sponsored by the National Aeronautics and Space Administration Contract NASw-905. 


$$
\mathcal{E}\left\{N^{2}\right\}=\sqrt{ } \lambda_{2} T / \pi
$$

$$
+\frac{2}{\pi^{2}} \int_{0}^{T}(T-\tau) \frac{M_{34}}{\left(1-r^{2}(\tau)\right)^{3 / 2} \Delta}\left[1+\Delta \tan ^{-1} \Delta\right] d \tau,
$$

where $M_{i j}$ is the cofactor of the ijth element of $\Sigma$ and $\Delta=M_{34} /\left(M_{33}^{2}-M_{34}^{2}\right)^{1 / 2}$. The dependence of $M_{34}$ and $\Delta$ on $\tau$ is suppressed in equation 2.

Indication of Proof. For convenience take $T=1$. Let $y_{n}(t)$ be a sequence of processes defined (as in [1]) to be equal to $x(t)$ at points of the form $t=k / 2^{n}, k=0,1, \cdots, 2^{n}, n=1,2, \cdots$, and linear between such points. Let $N_{n}$ denote the number of zeros of $y_{n}(t)$ in $0 \leqq t \leqq 1$. Then $N_{n} \uparrow N$ with probability one and, hence, $\mathcal{E}\left\{N_{n}\right\} \rightarrow \mathcal{E}\{N\}$, $\mathcal{E}\left\{N_{n}^{2}\right\} \rightarrow \mathcal{E}\left\{N^{2}\right\}$ as $n \rightarrow \infty$, by monotone convergence. But it follows by simple arguments that

$$
\begin{aligned}
\mathcal{E}\left\{N_{n}^{2}\right\}= & \varepsilon\left\{N_{n}\right\}+\lim _{e \rightarrow 0} \lim _{v \rightarrow \infty} \\
& \cdot \iint_{0 \leq s, t \leq 1 ;|t-s|>e} \varepsilon\left\{\delta_{v}\left(y_{n}(t)\right) \delta_{v}\left(y_{n}(s)\right)\left|y_{n}^{\prime}(t) \| y_{n}^{\prime}(s)\right|\right\} d t d s,
\end{aligned}
$$

where $\delta_{v}(x)=(v / \sqrt{ }(2 \pi)) e^{-v^{2} x^{2} / 2}$. The region of integration can be divided into three parts, $S_{1}, S_{2}, S_{3}$, where $s, t$ are in the same interval of the form $I_{n k}=\left[k / 2^{n},(k+1) / 2^{n}\right)$, adjacent intervals, or are separated by at least one such interval, respectively. It may be shown (by considerable calculation) that the first and second of these contributions vanish as $n \rightarrow \infty$, whereas the contribution from $S_{3}$ is

$$
\iint_{S_{\mathbf{3}}} d s d t \iint_{-\infty}^{\infty}|x y| p_{n, t, 8}(0,0, x, y) d x d y,
$$

in which $p_{n, t, s}(u, v, x, y)$ is the joint density for $y_{n}(t), y_{n}(s), y_{n}^{\prime}(t)$, $y_{n}^{\prime}(s)$. Further arguments, involving dominated convergence as $n \rightarrow \infty$, lead to the result (1), and (2) is obtainable from (1) by direct evaluation.

We note that the condition of the theorem cannot be significantly weakened. For it is possible to find a covariance function $r(\tau)$ for which

$$
\lambda_{2}+r^{\prime \prime}(\tau) \sim K /|\log | \tau|| \text { as } \tau \rightarrow 0 .
$$

This covariance function just fails to satisfy the integrability condition required for $\Psi$ and it can easily be shown that the second moment $\varepsilon\left\{N^{2}\right\}=\infty$. 
Finally, we note that it is apparent that the method can be used for crossings of any level $a$ and presumably for higher moments. However, in such cases the formulae rapidly become intractable.

\section{REFERENCES}

1. M. R. Leadbetter, On crossings of arbitrary curves by certain Gaussian processes, Proc. Amer. Math. Soc. 16 (1965), 60-68.

2. Yu. A. Rozanov and V. A. Volkonskii, Some limit theorems for random functions, Theor. Probability Appl. 5 (1961), 186-199.

3. H. Steinberg, P. M. Schultheiss, C. A. Wogrin and F. Zweig, Short time frequency measurements of narrow-band random signals by means of a zero counting process, J. Appl. Phys. 26 (1955), 195-201.

4. N. D. Ylvisaker, On level crossings of stationary Gaussian processes, University of Washington Technical Report No. 2, N.S.F. Grant G-25211, 1963.

RESEARCH TRIANGLE INSTITUTE AND

California Institute of Technology 